\title{
An Automated Computer Aided Procedure for Exploded View Generation
}

\author{
G.V.S.S. Sharma* and M.V.A. Raju Bahubalendruni \\ Mechanical Engineering Department, GMR Institute of Technology, GMR Nagar, Rajam-532127, Andhra Pradesh State, India
}

\begin{abstract}
Exploded view of a product is used in the applications for understanding the intricate details of the assembly. The problem with all the present-day CAD software packages is that the reconciliation of the product assembly from the exploded view is performed inaccurately wherein the parts collide with one another and the original assembly is not regained back through collision free path thereby leading to non-optimal performability of the software package. This present work proposes an efficient process for successfully generating the exploded view for assemblies through collision free paths, so that the reconciliation of product assembly from exploded view is accurate. The technique adopted here is based on the assembly coherence data and disassembly feasibility testing and is proposed in the context of a computer aided geometric assembly of a knuckle joint and internal combustion engine connecting rod assembly
\end{abstract}

Keywords: Assembly coherence; disassembly feasibility; product exploded view; CAD geometric model.

(Submitted on March 11, 2017; Revised on July 4, 2017; Accepted on July 6, 2017)

(C) 2017 Totem Publisher, Inc. All rights reserved.

\section{Introduction}

Any machine or mechanism constitutes individual parts assembled to form a wholesome and complete product. During the assemblage of individual parts, firstly the part forming the core or centre of the assembly is assembled first and the remaining parts are joined to this central part. With the advent of computers, geometric Computer Aided Design (CAD) models have revolutionized the way of visualizing the product assemblies. Exploded views have paved way for detailed visualization of the individual components constituting the assembly as observed by Brumbach and Clade [1]. The implementation of exploded view can be dated back to early fourteenth century in the works of Marino Taccola, an Italian mathematician, artist and engineer of early Renaissance period, as noted by Ferguson [2]. The usage of exploded views were further improved by Francesco di Giorgio and Leonardo da Vinci in fifteenth century, as noted by Ferguson [2]. Leonardo was the first person to create and comprehend the exploded views of a reciprocating motion machine. The concept of exploded view was further extended to human anatomy by Leonardo which was revealed by [3]. Sharma and Dumpala [4] studied the pedagogical applications of spatial visualization capability. The domain of visualization of the exploded components of an assembly demands spatial visualization capability and thorough knowledge of the assemblage.

\footnotetext{
* Corresponding author.

E-mail address: sarma.gvss@gmail.com
} 
In recent times, the product catalogues and machine manuals contain the exploded views which are often blurred and not clear, leading to excess visual distraction as observed by Li et al. [5]. The main issue of concern in generating an exploded view of a CAD geometric model is that during the backward integration of the exploded view, the individual part models collide each other and do not trace a collision free path to from the original assembly.

Thus, this work addresses this lacuna of generating back an assembly from the exploded view through proper sequence so that the parts do not collide with each other. This aspect of the CAD geometric modelling is known as assembly sequence planning as detailed by various authors $[6,7,8,9,14]$, certified that the correct sequence of assembly and disassembly operations requires the complete knowledge of assembly coherence data, geometric feasibility and stability information. Although there exists vast literature pertinent to application of artificial intelligence techniques and assembly knowledge database, the aspect of exploded view generation forms a neglected area without assigning due consideration. This research gap is pertinent in the works by various authors $[10,11,12,13,14,15]$.

At the outset, in the present research, a methodology is developed to generate exploded view automatically by making use of similarities between Assembly Sequence Planning (ASP) and exploded view as referred in works by Gu and Yan [16] and Castro [17]. This is achieved through assembly coherence information and geometric feasibility.

\section{Methodology}

The methodology traced in this work for achieving a collision free disassembly in a CAD geometric modelling software is delineated in the following steps:

Step-1: Formulate the assembly coherence matrix based on the surface or axial contact between the parts

Step-2: Based on the assembly coherence matrix trace out the pairs of parts in contact

Step-3: Locate the normal vector for disassembly feasibility of the part pairs

Step-4: Generate the disassembly feasibility square matrix of order $y_{i} \times x_{i}$ where " $y$ " represents the columns and " $x$ " represents the rows and number of columns and rows are equal for a square matrix. Here " $\mathrm{i}$ " represent the number of parts constituting the assembly.

Step-5: Identify the feasibility matrix column with $\sum y_{i}=(n-1)$, where $\sum y_{i}$ is the value of sum of a column and " $n$ " is the order of matrix

Step-6: Eliminate $\mathrm{y}_{\mathrm{i}}$ column and corresponding $\mathrm{x}_{\mathrm{i}}$ row to obtain the next level of feasible collision free disassembly of parts

Step-7: Repeat Step-5 and Step-6 till all the parts are assembled without colliding each other

\section{Case - 1: Knuckle joint}

\subsection{Assembly coherence information}

The assembly of any product consists of number of parts in contact with each other. All these individual parts form surface contact with each other. This surface contact of one part with another can be represented in a square matrix form where the size of the matrix equals to the total number of parts in the product assembly. This information, which gives the surface contact relations between each pair of parts, is known as the Assembly Coherence Information. In the CAD software products, the assembly coherence information is extracted by making use of the product design capabilities of the CAD software as evident in the work by Linn and Liu [18]. In the present case study of knuckle joint, Figure 1 shows the assembly of knuckle joint and Figure 2 shows the assembly coherence matrix. In the assembly coherence matrix the value " 0 " corresponds to no contact and the value " 1 " corresponds to a surface or axial contact among the corresponding part of that particular row and column of the assembly coherence matrix. 


\subsection{Disassembly feasibility aspect}

While disassembling an assembly in any CAD geometric modelling software, it is observed that the parts collide with each other while disassembling. This is identified as a research gap and an attempt has been made to derive a collision free path while disassembling the parts of an assembly. From the assembly coherence matrix in Figure 2, the part assembly pairs possessing the value of " $l$ ", form the part pairs is the subject of study. As such, the part pairs identified for this knuckle joint case study are: A-C; A-D, B-C, C-D, C-E, D-E and A-B. These are the only assembly pairs forming a physical contact with each other.

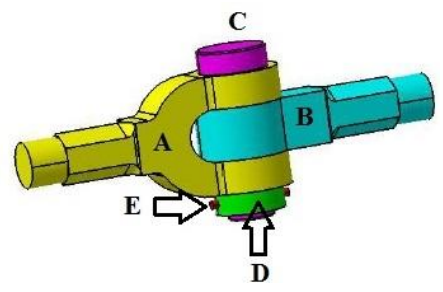

$$
\begin{aligned}
& \text { A }=\text { Fork end } \\
& \mathbf{B}=\text { Eye } \\
& \mathbf{C}=\text { Knuckle pin } \\
& \mathbf{D}=\text { Collar } \\
& \mathbf{E}=\text { Taper pin }
\end{aligned}
$$

\begin{tabular}{|c|c|c|c|}
\hline & $A \quad B$ & $B \quad C$ & $D$ \\
\hline$A$ & {$\left[\begin{array}{ll}0 & 1\end{array}\right.$} & 11 & 10 \\
\hline & 10 & $0 \quad 1$ & $0 \quad 0$ \\
\hline & $\begin{array}{ll}1 & 1\end{array}$ & 10 & 11 \\
\hline & 10 & $0 \quad 1$ & $\begin{array}{ll}0 & 1\end{array}$ \\
\hline & {$\left[\begin{array}{ll}0 & 0\end{array}\right.$} & $0 \quad 1$ & 10 \\
\hline
\end{tabular}

Figure 1. Assembly of knuckle joint

Figure 2. Assembly coherence matrix for knuckle joint

\subsubsection{Identification of feasibility vector}

\begin{tabular}{|c|c|c|c|c|}
\hline $\begin{array}{l}\text { PART } \\
\text { PAIR }\end{array}$ & $\begin{array}{l}\text { COORDINATES } \\
\text { ORIENTATION }\end{array}$ & $\begin{array}{c}\text { MATING PARTS \& } \\
\text { SURFACES }\end{array}$ & $\begin{array}{c}\text { NORMAL } \\
\text { REPRESENTATION } \\
\end{array}$ & VECTOR \\
\hline$A-C$ & & A & & $\begin{array}{l}N_{4}(0,0,1) \\
N_{5}(0,0,-1)\end{array}$ \\
\hline A-D & & & & $\begin{array}{l}N_{4}(0,0,1) \\
N_{5}(0,0,-1)\end{array}$ \\
\hline B-C & & & & $\begin{array}{c}N_{4}(0,0,1) \\
N_{5}(0,0,-1)\end{array}$ \\
\hline & & & & \\
\hline
\end{tabular}

After identifying the part pairs, the direction normal to the contact surface is identified as the normal vector and possibility of disassembling the part pairs along these normal vectors is checked. The part pairs, coordinate orientation, mating parts and surfaces, normal representation and the corresponding vector are shown in Table 1.

Table 1: Vectorial representation of part pair surfaces of knuckle joint assembly 


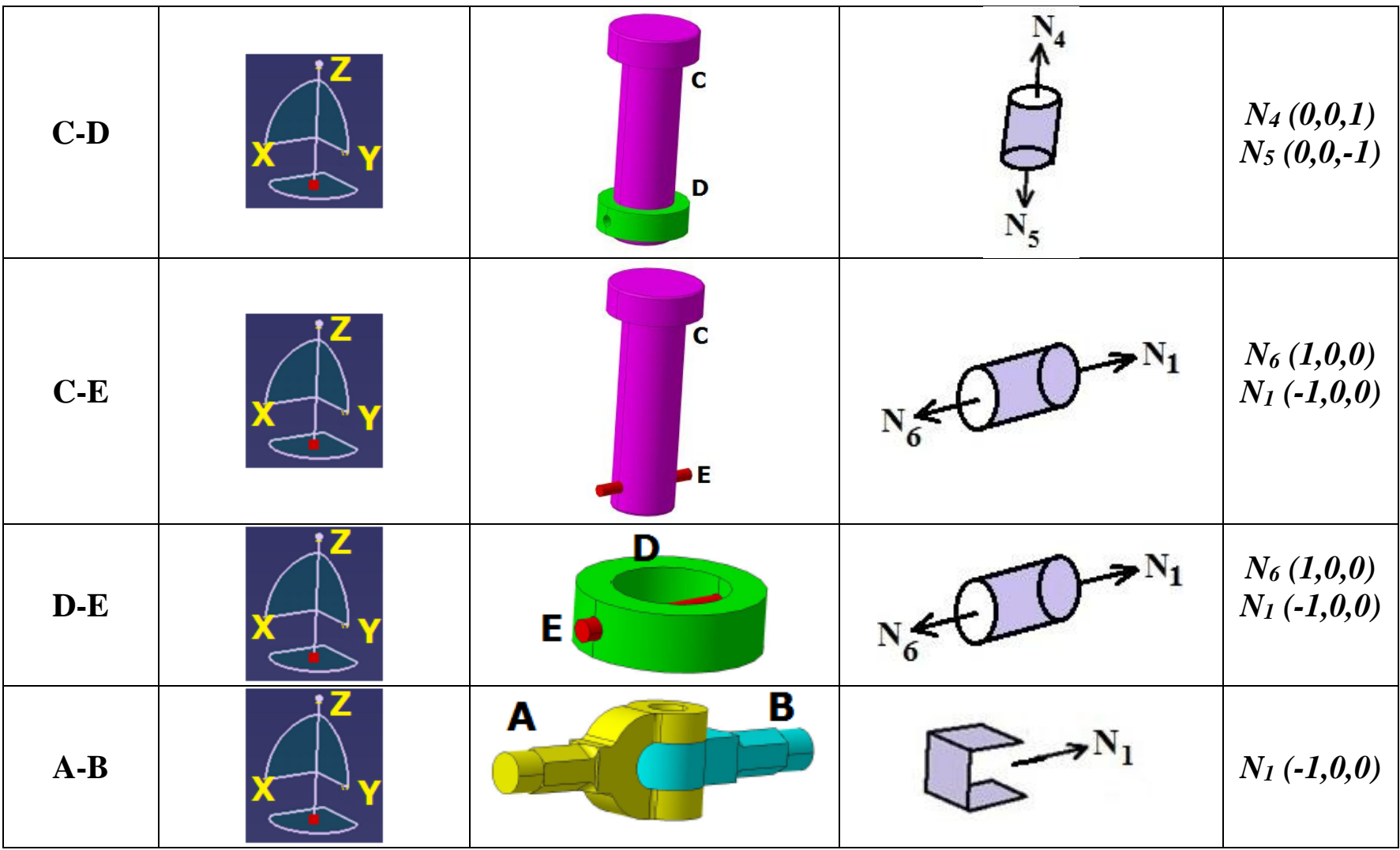

\subsubsection{Feasibility vector matrix}

In the present case study of knuckle joint, there are 5 components constituting the assembly, namely, the Fork end, Eye, Knuckle pin, Collar and Taper pin, which are labelled as parts A,B,C,D and E respectively. Hence, the feasibility vector matrix of disassembly level 1 is a square matrix of the order of $5 \times 5$. Here, the $\mathrm{n}=5$, where $\mathrm{n}$ represents the order of a square matrix. If the sum of individual columns equals to one less than the order of matrix, i.e., if sum of values in a column is equal to (n-1), then the part corresponding to that column can be removed in the corresponding normal direction, in the presence of all other parts. Taking this condition into account, the feasibility vector matrices for Disassembly level -1 is generated and shown in Table 2.

In the disassembly level 1, no part can be disassembled in $\mathrm{N} 1$ direction in the presence of other parts because none of the values of, sum of individual column, is equal to $(n-1)=(5-1)=4$. The same applies for the N4 and N5 directions. Whereas in N6 direction the part $\mathrm{E}$ can be disassembled in the presence of all other parts, because the sum of values in column $\mathrm{E}$ is equal to $(\mathrm{n}-1)=(5-1)=4$.

Table 2: Disassembly Level - 1 for knuckle joint

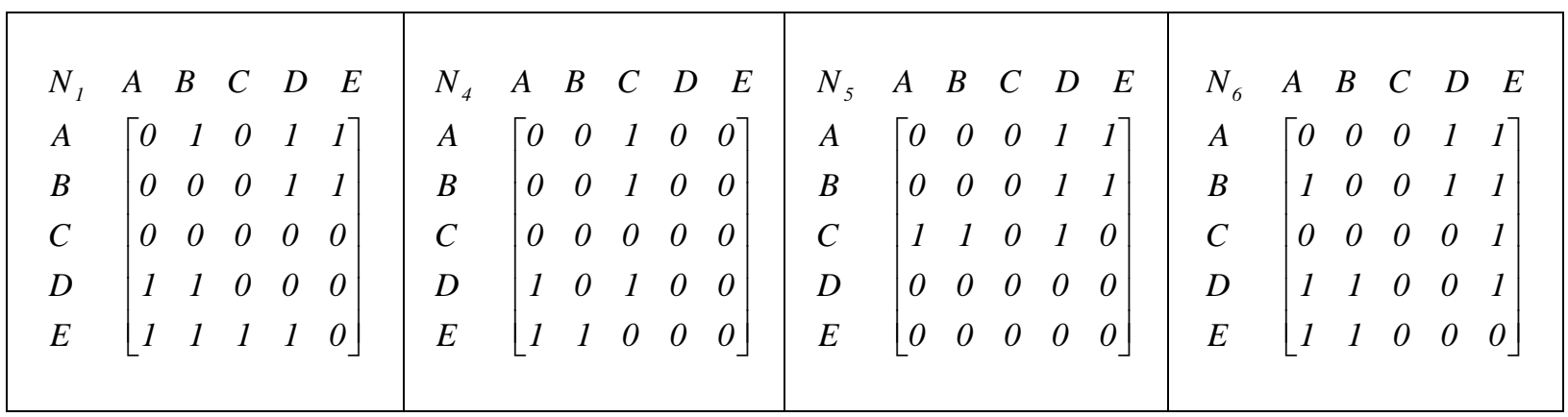


Hence, we eliminate the row and column pertaining to part E in order to obtain the disassembly level 2 . This leads to a square matrix of order, $n=4$, in the disassembly level 2. The parts disassembled after level -1 are depicted in the Figure 3 as shown below.

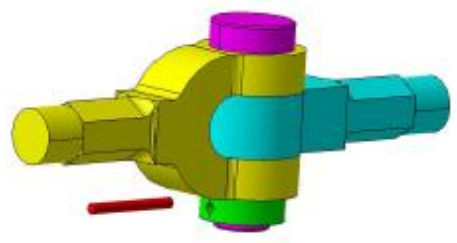

Figure 3: Level-1 disassembly of knuckle joint

After eliminating the row and column corresponding to part E, we obtain the Level-2 feasibility vector matrices as shown in Table 3. In the Level 2 , we obtain a feasibility vector square matrix of order $4 x 4$. Hence, $n=4$ and considering the previous condition that if the sum of individual columns equals to one less than the order of matrix, i.e., if sum of values in a column $=(\mathrm{n}-1)$, then, the part corresponding to that column can be removed in the corresponding normal direction, in the presence of all other parts. Taking this condition into account, the feasibility vector matrices for Disassembly level -2 is generated.

Table 3: Disassembly Level - 2 for knuckle joint

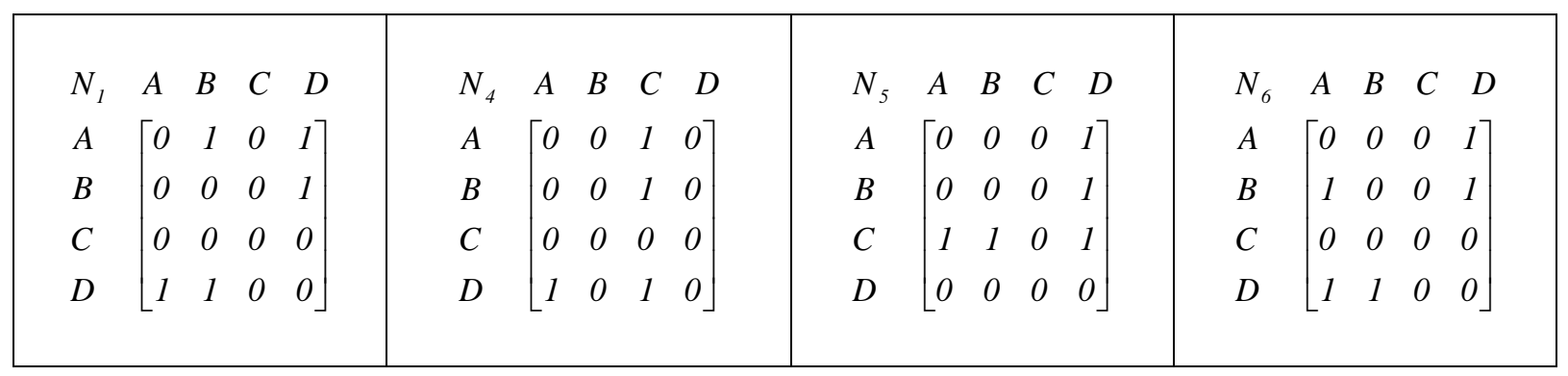

In this disassembly level 2, no part can be disassembled in N1 and N6 directions in the presence of other parts because none of the values of, sum of individual column, is equal to $(n-1)=(4-1)=3$. In N4 direction the part $\mathrm{C}$ can be disassembled in the presence of all other parts, because the sum of values in column $C$ is equal to $(n-1)=(4-1)=3$. Hence, we eliminate the row and column pertaining to part $\mathrm{C}$ in order to obtain the disassembly level 3 . In N5 direction the part $\mathrm{D}$ can be disassembled in the presence of all other parts, because, the sum of values in column D is equal to $(n-1)=(4-1)=3$.

Hence, we eliminate the row and column pertaining to part D in order to obtain the disassembly level 3. The parts disassembled after level -2 are depicted in the Figure 4 as shown below.

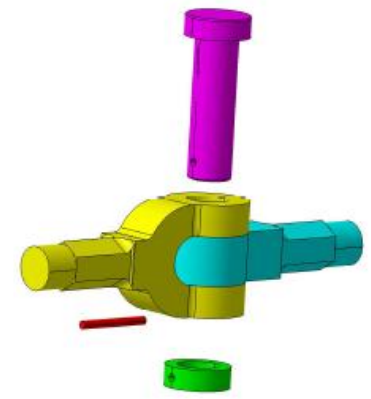

Figure 4: Level-2 disassembly of knuckle joint

After eliminating the row and column corresponding to part C and part D, we obtain the Level-3 feasibility vector matrices of order $2 \times 2$ as shown in Table 4. Hence, $n=2$ and considering the previous condition that if the sum of individual columns equals to one less than the order of matrix, i.e., if sum of values in a column is equal to (n-1), then, the part corresponding to 
that column can be removed in the corresponding normal direction, in the presence of all other parts. Taking this condition into account, the feasibility vector matrices for Disassembly level -3 is generated.

Table 4: Disassembly level - 3 for knuckle joint

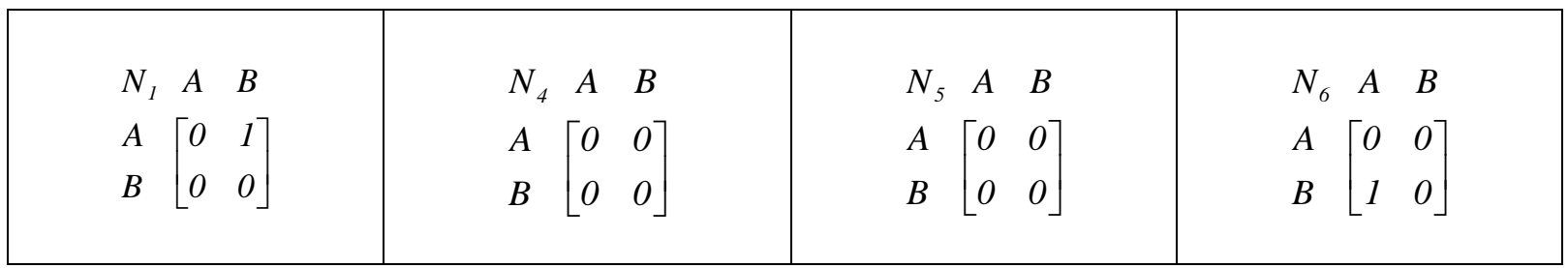

In disassembly level 3, the part B can be disassembled in N1 direction and part A can be disassembled in N6 direction in the presence of all other parts, because, the sum of values in column B is equal to $(n-1)=(2-1)=1$ and the same applies for column A also. Whereas in N4 and N5 directions none of the values of, sum of individual column, is equal to $(n-1)=(2-1)=$ 1 and hence no part can be disassembled in N4 and N5 directions in the presence of other parts.

The parts disassembled after level -3 are depicted in the Figure 5 as shown below. This completes the collision free exploded view of the complete knuckle joint assembly.

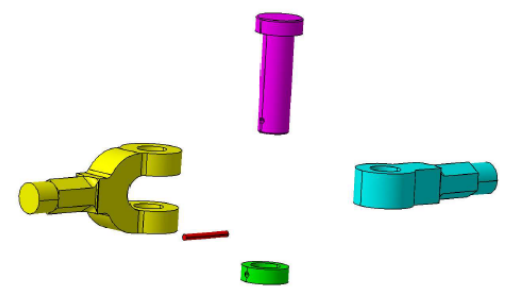

Figure 5: Level-3 disassembly of knuckle joint

\section{Case - 2: Connecting rod}

In the case study of connecting rod assembly, Figure 6 depicts the assemblage of connecting rod along with the part nomenclature. The assembly coherence information is captured in the form of assembly coherence matrix in Figure 7.

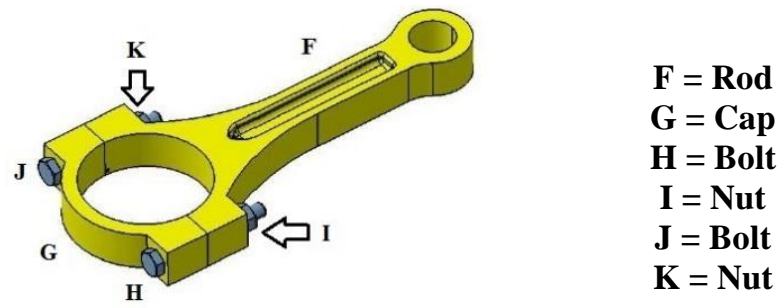

Figure 6: Assembly of connecting rod

$F$
$F$
$G$
$H$
$H$
$I$
$J$
$K$
$K$$\left[\begin{array}{lllllll}0 & 1 & 1 & 1 & 1 & 1 \\ 1 & 0 & 1 & 0 & 1 & 0 \\ 1 & 1 & 0 & 1 & 0 & 0 \\ 1 & 0 & 1 & 0 & 0 & 0 \\ 1 & 1 & 0 & 0 & 0 & 1 \\ 1 & 0 & 0 & 0 & 1 & 0\end{array}\right]$

Figure 7: Assembly coherence matrix for connecting rod

The disassembly feasibility aspect focuses on the identification of part pairs, mating surfaces, normal representation of mating surfaces and the disassembly feasibility vectors. This information is captured in Table 5 . 
Table 5: Vectorial representation of part pair surfaces of connecting rod assembly

\begin{tabular}{|c|c|c|c|c|}
\hline $\begin{array}{l}\text { PART } \\
\text { PAIR }\end{array}$ & $\begin{array}{l}\text { COORDINATES } \\
\text { ORIENTATION }\end{array}$ & $\begin{array}{c}\text { MATING PARTS \& } \\
\text { SURFACES }\end{array}$ & $\begin{array}{c}\text { NORMAL } \\
\text { REPRESENTATION }\end{array}$ & VECTOR \\
\hline F-G & & & & $\begin{array}{l}N_{1}(1,0,0) \\
N_{2}(-1,0,0)\end{array}$ \\
\hline F-H & & & & $\begin{array}{l}N_{1}(1,0,0) \\
N_{2}(-1,0,0)\end{array}$ \\
\hline F-I & & & & $\begin{array}{l}N_{1}(1,0,0) \\
N_{2}(-1,0,0)\end{array}$ \\
\hline F-J & & & & $\begin{array}{l}N_{1}(1,0,0) \\
N_{2}(-1,0,0)\end{array}$ \\
\hline F-K & & & & $\begin{array}{l}N_{1}(1,0,0) \\
N_{2}(-1,0,0)\end{array}$ \\
\hline G-H & & & & $\begin{array}{l}N_{1}(1,0,0) \\
N_{2}(-1,0,0)\end{array}$ \\
\hline G-J & & & & $\begin{array}{l}N_{1}(1,0,0) \\
N_{2}(-1,0,0)\end{array}$ \\
\hline H-I & & & & $\begin{array}{l}N_{1}(1,0,0) \\
N_{2}(-1,0,0)\end{array}$ \\
\hline
\end{tabular}




J-K

In the present case study of connecting rod, there are 6 components constituting the assembly namely, the rod end, cap, bolt, nut, bolt and nut, which are labelled as parts F, G, H, I, J and K respectively. Hence, the feasibility vector matrix of disassembly level 1 is a square matrix of the order of $6 \times 6$. Here, the value of $n$ is equal to 6 where, $n$ represents the order of a square matrix. If the sum of individual columns equals to one less than the order of matrix, i.e., if sum of values in a column is equal to (n-1), then the part corresponding to that column can be removed in the corresponding normal direction, in the presence of all other parts. Taking this condition into account, the feasibility vector matrices for Disassembly level -1 is generated and shown in Table 6.

Table 6: Disassembly Level - 1 for connecting rod

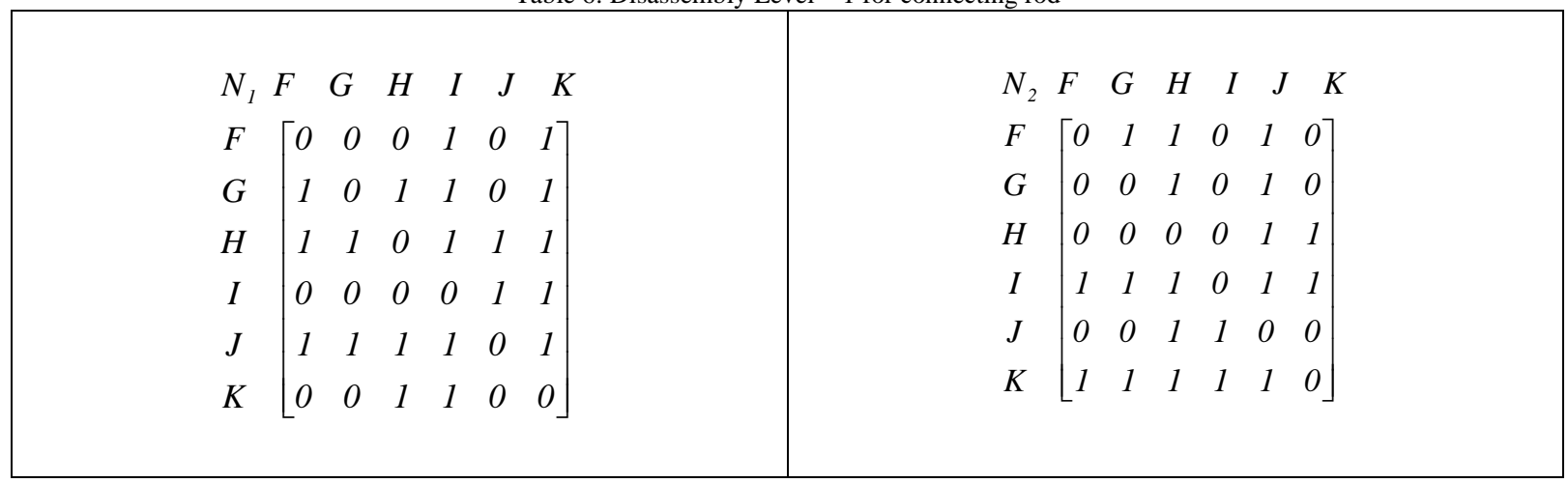

In this disassembly level 1 , the parts I and $\mathrm{K}$ can be disassembled in $\mathrm{N}_{1}$ direction in the presence of all other parts, because the sum of values in column $I$ and the sum of values in column $I$ and $K$ is individually equal to $(n-1)=(6-1)=5$. Also here the parts $\mathrm{H}$ and $\mathrm{J}$ can be disassembled in $\mathrm{N} 2$ direction in the presence of all other parts, because, the sum of values in column $\mathrm{H}$ and $J$ is equal to $(n-1)=(6-1)=5$. Hence, we eliminate the row and column pertaining to parts $I, K, H$ and $J$ in order to obtain the disassembly level 2 . This leads to a square matrix of order, $n=2$, in the disassembly level 2 . The parts disassembled after level -1 are depicted in the Figure 8 as shown below.

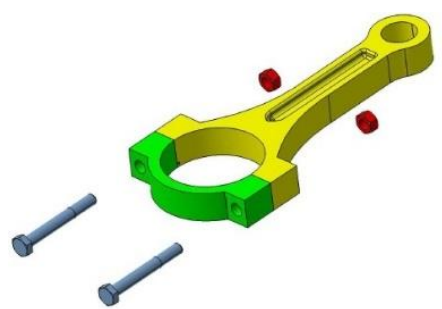

Figure 8: Level - 1 disassembly of connecting rod

After eliminating the rows and columns corresponding to parts I, K, H and J, we obtain the Level-2 feasibility vector matrices as shown in Table 7. In this disassembly level 2, the part F can be disassembled in the presence of all other parts in $\mathrm{N} 1$ direction, because the sum of values in column $\mathrm{F}$ is equal to $(\mathrm{n}-1)=(2-1)=1$. Similarly, as per the same condition, the part $\mathrm{G}$ can be disassembled in the presence of all other parts in $\mathrm{N} 2$ direction.

The parts disassembled after level -2 are depicted in the Figure 9 as shown below. This completes the collision free exploded view of the complete connecting rod assembly.

Table 7: Disassembly Level -2 for connecting rod

$$
\begin{aligned}
& N_{l} \quad F \quad G \\
& \begin{array}{l}
F \\
G
\end{array}\left[\begin{array}{ll}
0 & 0 \\
1 & 0
\end{array}\right] \\
& N_{2} \quad F \quad G \\
& F \quad\left[\begin{array}{ll}
0 & 1 \\
0 & 0
\end{array}\right]
\end{aligned}
$$




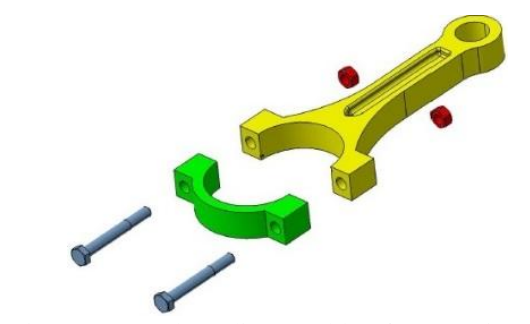

Figure 9: Level - 2 disassembly of connecting rod

\section{Conclusion}

An improved strategy for the exploded view of assemblages has been introduced by considering two different case studies of disassembly of knuckle joint and connecting rod assemblages. This enhanced exploded view generation method takes into consideration, the assembly coherence information, disassembly feasibility aspect through identification of feasibility vectors and corresponding feasibility matrices. From this, different levels of disassembly are generated which paves way for a disassembly path where the parts do not collide with each other while generating the exploded views of the assemblage.

The proposed strategy is capable of solving canonical and non-canonical perspectives based on the mating surfaces. At the outset, the present method offers a coherent and collision free path for generating exploded view of a CAD geometric model, which eases the process of disassembly of a product and also clarifies the spatial visualization of the geometric model and ultimately improves the performability of CAD software package.

\section{References}

1. M. Brumbach, J. Clade, "Industrial Maintenance", Nelson Education, 2013.

2. $\quad$ E.S. Ferguson, "Engineering and the Mind's Eye", MIT press, 1994.

3. D. Laurenza, M. Taddei, E. Zanon, "Leonardo's Machines: Da Vinci's Inventions Revealed”, David \& Charles, 2006.

4. G.V.S.S. Sharma, Ravikumar Dumpala, "Teaching of Mechanical Engineering Concepts through Three-Dimensional Geometric Modeling”, International Journal of Mechanical Engineering Education vol. 43, no.3, pp.180-190, 2015.

5. W. Li, M. Agrawala, B.Curless, D. Salesin, "Automated Generation of Interactive 3D Exploded View Diagrams", ACM Transactions on Graphics (TOG), vol. 3, pp. 101. ACM, 2008.

6. D.E. Whitney, "Mechanical Assemblies: Their Design, Manufacture, and Role in Product Development", vol. 1, Oxford University Press on Demand, 2004.

7. L.H. De Mello, A.C. Sanderson, "A Correct and Complete Algorithm for the Generation of Mechanical Assembly Sequences", IEEE transactions on Robotics and Automation, vol. 7, no.2, pp.228-240, 1991.

8. M.R. Bahubalendruni, B.B. Biswal, "A Review on Assembly Sequence Generation and Its Automation", Proceedings of the Institution of Mechanical Engineers, Part C: Journal of Mechanical Engineering Science, vol. 230, no.5, pp. 824-838, 2016a.

9. M.R. Bahubalendruni, B.B. Biswal, M. Kumar, R. Nayak, "Influence of Assembly Predicate Consideration on Optimal Assembly Sequence Generation”, Assembly Automation, vol. 35, no.4, pp.309-316, 2015 a.

10. H.R. Lee, D.D. Gemmill, "Improved Methods of Assembly Sequence Determination for Automatic Assembly Systems", European journal of operational research, vol. 131, no.3, pp. 611-621, 2001.

11. R. Ben Hadj, M. Trigui,N. Aifaoui, "Toward an Integrated CAD Assembly Sequence Planning Solution", Proceedings of the Institution of Mechanical Engineers, Part C: Journal of Mechanical Engineering Science, vol. 229, no.16, pp.2987-3001, 2015.

12. M.R. Bahubalendruni, B.B. Biswal, "Liaison Concatenation-A Method to Obtain Feasible Assembly Sequences from 3D-CAD Product”, Sadhana vol. 41, no.1, pp.67-74, 2016b.

13. M.R. Bahubalendruni, B. Deepak, B.B. Biswal, "An Advanced Immune based Strategy to Obtain an Optimal Feasible Assembly Sequence”, Assembly Automation, vol. 36, no.2, pp. 127-137, 2016c.

14. M.R. Bahubalendruni, B.B Biswal, "A Novel Concatenation Method for Generating Optimal Robotic Assembly Sequences". Proceedings of the Institution of Mechanical Engineers, Part C: Journal of Mechanical Engineering Science, 0954406215623813,2015 b.

15. M.V.A.Bahubalendruni, B. Biswal, V. BB, "Optimal Robotic Assembly Sequence Generation using Particle Swarm Optimization”, Journal of Automation and Control Engineering, vol. 4, no.2, 2015c.

16. P. Gu, X. Yan, "CAD-Directed Automatic Assembly Sequence Planning”, International Journal of Production Research, vol. 33, no.11, pp. 3069-3100, 1995.

17. C.G. Castro, "Comportamento Mecânico e Biológico de Implante Dentário Com Interface Cônica Interna”, 2015.

18. R.J. Linn, H. Liu, “An Automatic Assembly Liaison Extraction Method and Assembly Liaison Model," IIE transactions, vol. 31, no. 4, pp. 353-363, 1999. 
G. V. S. S. Sharma is presently working as an Assistant Professor in the Department of Mechanical Engineering, GMR Institute of Technology Rajam, an autonomous institute affiliated to JNTU Kakinada, located in Srikakulam, Andhra Pradesh, India. He has obtained his first Postgraduation certificate from Central Institute of Tool Design, Hyderabad, India in the field of Tool, Die and Mould Design. He also specialized in CAD/CAM as his second Postgraduation M.Tech degree from GMRIT, rajam, India. He obtained his bachelor's degree in Mechanical Engineering from Swami Ramanand Teerth Marathwada University, Nanded, India. He has work experience of 15 years in various capacities in the field of Indian automobile industry and engineering education.

M. V. A. Raju Bahubalendruni is currently working as an Associate Professor in Department of Mechanical Engineering at GMRIT, Rajam, and Andhra Pradesh and has vast teaching and industry experience. He received his Ph.D. from National Institute of Technology, Rourkela, India in 2017. His research interest includes Composite Materials, Assembly Automation. He has published two patents and has published papers also in peer-refereed journals and he is reviewer (SCI) for Assembly Automation (10), Proceedings of the Institution of Mechanical Engineers, Part B: Journal of Engineering Manufacture (10) attended many International Conferences. 